\title{
Global Output Feedback Stabilization of Nonlinear Systems with a Time-Varying Power and Unknown Output Function
}

\author{
Chao Guo ${ }^{1}$ and Kemei Zhang $\mathbb{D}^{2}$ \\ ${ }^{1}$ Institute of Automation, Qufu Normal University, Shandong Province 273165, China \\ ${ }^{2}$ School of Mathematics Sciences, Qufu Normal University, Shandong Province 273165, China \\ Correspondence should be addressed to Kemei Zhang; zhkm90@126.com
}

Received 24 June 2018; Accepted 10 September 2018; Published 27 September 2018

Guest Editor: Weihai Zhang

Copyright (c) 2018 Chao Guo and Kemei Zhang. This is an open access article distributed under the Creative Commons Attribution License, which permits unrestricted use, distribution, and reproduction in any medium, provided the original work is properly cited.

This paper studies the problem of global output feedback stabilization for a class of nonlinear systems with a time-varying power and unknown output function. For nonlinear systems with a time-varying power and unknown continuous output function, by constructing a new nonlinear reduced-order observer together with adding a power integrator method, a new function to determine the maximal open sector $\Omega$ of output function is given. As long as output function belongs to any closed sector included in $\Omega$, it is shown that the equilibrium point of the closed-loop system can be guaranteed globally uniformly asymptotically stable by an output feedback controller.

\section{Introduction}

Consider nonlinear systems with the unknown output function

$$
\begin{aligned}
& \dot{x}_{i}(t)=\left[x_{i+1}(t)\right]^{p(t)}+\phi_{i}\left(t, x_{1}(t), \ldots, x_{i}(t)\right), \\
& i=1, \ldots, n-1, \\
& \dot{x}_{n}(t)= {[u(t)]^{p(t)}+\phi_{n}\left(t, x_{1}(t), \ldots, x_{n}(t)\right), } \\
& y(t)=h\left(x_{1}(t)\right),
\end{aligned}
$$

where $x=\left(x_{1}, \ldots, x_{n}\right)^{\top} \in R^{n}, u \in R$, and $y \in R$ are the unmeasurable state, control input, and output, respectively. For a real constant $\varrho>0,[\cdot]^{\varrho}$ is defined as $[\cdot]^{\varrho}=\operatorname{sgn}(\cdot)|\cdot|^{\varrho}$. The time-varying power $p(t): R^{+} \longrightarrow R^{+}$is a continuous bounded function satisfying $1 \leq \underline{p} \leq p(t) \leq \bar{p}$ with two constants $p$ and $\bar{p}$. For $i=1, \ldots, n, \phi_{i}(\cdot): R^{+} \times R^{i} \longrightarrow R$ are continuous in the first argument and locally Lipschitz with respect to the rest variables with $\phi_{i}(t, 0)=0$. Output function $h(\cdot)$ is an unknown continuous function with $h(0)=0$.
Over the past decade, with the help of adding a power integrator method, homogeneous domination method, and recursive observer design, there exist some interesting results on state/output feedback design of high-order nonlinear systems, whose powers are known constant ratios of odd integers; see [1-16] and the references therein.

In recent years, some interesting results have been achieved on output feedback design of nonlinear systems with known constant powers and unknown output function. For the nonlinear systems (1) with $p(t)=1$, when $h(\cdot)$ is a continuous differentiable function and its derivative with known upper and lower bounds, global output feedback stabilization and finite-time output feedback stabilization have been achieved in [17-19] and [20], respectively. When the derivative of $h(\cdot)$ is with unknown upper bound, [21] achieved semi-global output feedback control. Furthermore, when $h(\cdot)$ is extended to be only a continuous function, with the help of the given maximal sector region of output function, [22] achieved output feedback stabilization. Lately, in [23], a new design and analysis method for high-order nonlinear systems with unknown continuous output function was proposed based on adding 
a power integrator method and homogeneous domination method.

According to some practice [24-26], it is well-known that the timely deteriorated performance of system often results in different running data, which usually identify the powers of system. Therefore, the powers of system are usually not fixed and can be varying with a suitable bound even in the same working condition. For example, as a practical second-order dynamic model of reduced-order boiler-turbine unit, two typical different powers $p(t)=1.072$ and $p(t)=1.031$ have been identified in [25] and [26], respectively. For nonlinear systems with a time-varying power, $[27,28]$ achieved global state feedback stabilization based on interval homogeneous domination approach. As far as we know, [29] is the first paper to study the output feedback stabilization of nonlinear systems (1) with $h\left(x_{1}\right)=x_{1}$ by the revamped method of adding a power integrator together with the recursive nonlinear observer design. Naturally, an interesting problem is put forward: For more general nonlinear systems (1) with $h\left(x_{1}\right)$ being an unknown continuous function, can we design an output feedback controller?

In this paper, we make an attempt to handle this problem. Some essential technical difficulties in control design will be inevitably produced: (i) Compared with [29], since output function is unknown, we construct a new nonlinear reducedorder observer without using the unmeasurable state $x_{1}$. (ii) Compared with [23], a new function to determine the maximal open sector $\Omega$ of output function is given since the power of system is time-varying. As long as output function belongs to any closed sector included in $\Omega$, the equilibrium point of the closed-loop system is globally uniformly asymptotically stable under the constructed output feedback controller.

This paper is organized as follows. Section 2 gives some preliminaries. The design and analysis of output feedback controller are given in Section 3, following a simulation example in Section 4. Section 5 concludes this paper.

\section{Mathematical Preliminaries}

Some notations, definitions, and lemmas are to be used throughout this paper.

In this paper, the argument of function will be omitted whenever no confusion can arise from the context. $R, R^{+}$, and $R^{n}$ denote the set of real numbers, the set of all nonnegative real numbers, and the real $n$-dimensional space. For constant $\beta_{i}, i=1, \ldots, n$, let $\beta_{i \sim j}=\prod_{k=i}^{j} \beta_{k}, 1 \leq i<j \leq n$, and $\beta_{i \sim i}=$ $\beta_{i}, \beta_{i \sim(i-1)}=1, \beta_{0 \sim i}=0$. For integers a, $b$ and constant $\Lambda_{i}$, $\sum_{i=a}^{b} \Lambda_{i}=0$ when $a>b$.

Definition 1 (see [30]). A function $h(\cdot): R \longrightarrow R$ is said to belong to the sector $\left[\rho_{1}, \rho_{2}\right]$ if $\left(h(s)-\rho_{1} s\right)\left(h(s)-\rho_{2} s\right) \leq 0$, where $\rho_{1}$ and $\rho_{2}$ are constants with $\rho_{1}<\rho_{2}$. If the inequality is strict, we write the sector as $\left(\rho_{1}, \rho_{2}\right)$.

The following lemmas will serve as the basis for the development of output feedback controller. Lemmas 2-5 are used to enlarge inequalities. Lemmas 6 and 7 are Lyapunov stability theorem for the global uniformly asymptotically stable of the closed-loop system.

Lemma 2 (see [31]). Let $p(t)$ be a real-valued function of $t \in$ $R^{+}$satisfying $0<p(t)<+\infty$. For any $x_{i} \in R, i=1, \ldots, n$,

$$
\begin{aligned}
& \left(\left|x_{1}\right|+\cdots+\left|x_{n}\right|\right)^{p(t)} \\
& \quad \leq \max \left\{n^{p(t)-1}, 1\right\}\left(\left|x_{1}\right|^{p(t)}+\cdots+\left|x_{n}\right|^{p(t)}\right) .
\end{aligned}
$$

Lemma 3 (see [32]). Let $p(t), q(t)$ be positive real-valued functions of $t \in R^{+}$and $\gamma(x, y)$ be a positive real-valued function of $x, y \in R$. For any $x, y \in R$,

$$
\begin{aligned}
|x|^{p(t)}|y|^{q(t)} & \\
\leq & \frac{p(t)}{p(t)+q(t)} \gamma(x, y)|x|^{p(t)+q(t)} \\
& \quad+\frac{q(t)}{p(t)+q(t)} \gamma^{-p(t) / q(t)}(x, y)|y|^{p(t)+q(t)} .
\end{aligned}
$$

Lemma 4 (see [33]). Let $p(t)$ be a real-valued function of $t \epsilon$ $R^{+}$satisfying $p(t) \geq 1$. For any $x, y \in R$,

$$
\begin{aligned}
& \left|[x]^{p(t)}-[y]^{p(t)}\right| \\
& \quad \leq p(t)\left(2^{p(t)-3}+1\right)\left(|x-y|^{p(t)}+|x-y||y|^{p(t)-1}\right),
\end{aligned}
$$

where $\left|y^{p(t)-1}\right|:=0$ if $y=0$ and $p(t)=1$.

Lemma 5 (see [33]). Let $p(t)$ be a real-valued function of $t \in$ $R^{+}$satisfying $p(t) \geq 1$. For any $x, y \in R$,

$$
-(x-y)\left([x]^{p(t)}-[y]^{p(t)}\right) \leq-2^{1-p(t)}|x-y|^{p(t)+1} .
$$

Lemma 6 (see [30]). Let $V: R^{n} \longrightarrow R$ be a continuous positive definite and radially unbounded function defined on $R^{n}$; then there exist class $\mathscr{K}_{\infty}$ functions $\pi_{1}$ and $\pi_{2}$ defined on $[0,+\infty)$ such that $\pi_{1}(\|x\|) \leq V(x) \leq \pi_{2}(\|x\|)$ for all $x \in R^{n}$.

Lemma 7 (see [30]). For the nonautonomous system $\dot{x}=$ $f(t, x)$, let $x=0$ be an equilibrium point of system and $V: R^{+} \times R^{n} \longrightarrow R^{+}$be a continuously differentiable function such that $W_{1}(x) \leq V(t, x) \leq W_{2}(x)$ and $\partial V / \partial t+$ $(\partial V / \partial x) f(t, x) \leq-W_{3}(x)$ hold for any $t \geq 0$ and $x \in$ $R^{n}$, where $W_{1}(x), W_{2}(x), W_{3}(x)$ are continuous positive definite functions on $R^{n}$ and $W_{1}(x)$ is radially unbounded. Then $x=0$ is globally uniformly asymptotically stable.

\section{Output Feedback Controller Design and Stability Analysis}

3.1. Control Objective of This Paper. The objective of this paper is to construct an output feedback controller for system (1) such that the equilibrium point of the closed-loop system is globally uniformly asymptotically stable when the maximal open sector of output function is given. 
Assumption 8. There is a known constant $c \geq 0$ such that for $i=1, \ldots, n$,

$$
\begin{aligned}
& \left|\phi_{i}(t, x)-\phi_{i}(t, y)\right| \\
& \leq c \sum_{j=1}^{N}\left(\sum_{k=1}^{i}\left(\left|x_{k}-y_{k}\right|^{a_{k, j}(t)} \sum_{q=1}^{i}\left(\left|x_{q}\right|^{b_{k, j}(t)}+\left|y_{q}\right|^{b_{k, j}(t)}\right)\right)\right),
\end{aligned}
$$

for all $x, y \in R^{n}$, where $N$ is an arbitrary positive integer and the real-valued functions $a_{k, j}(t)$ and $b_{k, j}(t)$ satisfy the following relations:

$$
\begin{aligned}
& a_{k, j}(t)+b_{k, j}(t)=p(t), \\
& \underline{a}_{k, j} \leq a_{k, j}(t) \leq \bar{a}_{k, j}, \underline{b}_{k, j} \leq b_{k, j}(t) \leq \bar{b}_{k, j},
\end{aligned}
$$

for all $j=1, \ldots, N$ and $k=1, \ldots, i$, with $0<\underline{a}_{k, j} \leq \bar{a}_{k, j}$ and $0<\underline{b}_{k, j} \leq \bar{b}_{k, j}$. By Lemma 3 and Assumption 8, there exists a constant $\widetilde{c} \geq 0$ such that

$$
\begin{aligned}
&\left|\phi_{i}\left(t, x_{1}, \ldots, x_{i}\right)\right| \leq \tilde{c}\left(\left|x_{1}\right|^{p(t)}+\cdots+\left|x_{i}\right|^{p(t)}\right) \\
& \\
& i=1, \ldots, n .
\end{aligned}
$$

Remark 9. As far as we know, under Assumption 8, [29] is the first paper to study output feedback stabilization of nonlinear system (1) with $h\left(x_{1}\right)=x_{1}$. In this paper, we will consider system (1) with $h\left(x_{1}\right)$ being an unknown continuous function.

\subsection{State Feedback Controller Design of System (1)}

Step 1. Taking $\xi_{1}=x_{1}, V_{1}\left(x_{1}\right)=(1 / 2) x_{1}^{2}$, it follows from (1) and (8) that

$$
\begin{aligned}
\dot{V}_{1}= & x_{1}\left[x_{2}\right]^{p(t)}+x_{1} \phi_{1} \\
\leq & x_{1}\left(\left[x_{2}\right]^{p(t)}-\left[x_{2}^{*}\right]^{p(t)}\right)+x_{1}\left[x_{2}^{*}\right]^{p(t)} \\
& +\widetilde{c}\left|x_{1}\right|^{p(t)+1} .
\end{aligned}
$$

Choose the virtual controller $x_{2}^{*}=-\beta_{1} x_{1}$ with $\beta_{1}=\left(r_{1,1}+\right.$ $\widetilde{c})^{1 / \underline{p}}$, where $r_{1,1} \geq 1$ is a constant to be designed. Due to $\left(r_{1,1}+\right.$ $\widetilde{c})^{p(t) / \underline{p}} \geq\left(r_{1,1}+\widetilde{c}\right)$, (9) becomes

$$
\dot{V}_{1} \leq-r_{1,1}\left|\xi_{1}\right|^{p(t)+1}+\xi_{1}\left(\left[x_{2}\right]^{p(t)}-\left[x_{2}^{*}\right]^{p(t)}\right) .
$$

Inductive Step. Suppose that at step $k-1(k \geq 2)$, there is a positive definite and radially unbounded Lyapunov function $V_{k-1}\left(\xi_{1}, \ldots, \xi_{k-1}\right)=V_{k-2}\left(\xi_{1}, \ldots, \xi_{k-2}\right)+(1 / 2) \xi_{k-1}^{2}$ and a set of virtual controllers $x_{2}^{*}, \ldots, x_{k}^{*}$ defined by

$$
\begin{aligned}
\xi_{1} & =x_{1}, \\
x_{i}^{*} & =-\beta_{i-1} \xi_{i-1}, \\
\xi_{i} & =x_{i}-x_{i}^{*},
\end{aligned}
$$

$$
i=2, \ldots, k
$$

such that

$$
\begin{aligned}
\dot{V}_{k-1} \leq & -\sum_{i=1}^{k-2}\left(r_{i, i}-\tilde{r}_{i+1,1}-\sum_{j=i+1}^{k-1} \bar{r}_{j, i}\right)\left|\xi_{i}\right|^{p(t)+1} \\
& -r_{k-1, k-1}\left|\xi_{k-1}\right|^{p(t)+1} \\
& +\xi_{k-1}\left(\left[x_{k}\right]^{p(t)}-\left[x_{k}^{*}\right]^{p(t)}\right),
\end{aligned}
$$

where $\tilde{r}_{i+1,1}, \bar{r}_{j, i}, j=i+1, \ldots, k-1, i=1, \ldots, k-2$, are positive constants, $r_{1,1}, \ldots, r_{k-1, k-1} \geq 1$ are constants to be designed, and $\beta_{1}, \ldots, \beta_{k-1}$ are positive constants dependent on $r_{1,1}, \ldots, r_{k-1, k-1}$. In what follows, we will show that (12) still holds at step $k$.

By (11), one has $x_{k}^{*}=-\sum_{i=1}^{k-1} \beta_{i \sim(k-1)} x_{i}$, which together with (1) lead to

$$
\dot{\xi}_{k}=\left[x_{k+1}\right]^{p(t)}+\phi_{k}+\sum_{i=1}^{k-1} \beta_{i \sim(k-1)}\left(\left[x_{i+1}\right]^{p(t)}+\phi_{i}\right) .
$$

Constructing $V_{k}\left(\xi_{1}, \ldots, \xi_{k}\right)=V_{k-1}\left(\xi_{1}, \ldots, \xi_{k-1}\right)+(1 / 2) \xi_{k}^{2}$, it follows from (12) and (13) that

$$
\begin{aligned}
\dot{V}_{k} & \leq-\sum_{i=1}^{k-2}\left(r_{i, i}-\tilde{r}_{i+1,1}-\sum_{j=i+1}^{k-1} \bar{r}_{j, i}\right)\left|\xi_{i}\right|^{p(t)+1} \\
& -r_{k-1, k-1}\left|\xi_{k-1}\right|^{p(t)+1}+\xi_{k-1}\left(\left[x_{k}\right]^{p(t)}-\left[x_{k}^{*}\right]^{p(t)}\right) \\
& +\xi_{k}\left(\left[x_{k+1}\right]^{p(t)}+\phi_{k}\right. \\
& \left.+\sum_{i=1}^{k-1} \beta_{i \sim(k-1)}\left(\left[x_{i+1}\right]^{p(t)}+\phi_{i}\right)\right) .
\end{aligned}
$$

By (8), (11), and Lemmas 2-4, we derive

$$
\begin{aligned}
& \xi_{k-1}\left(\left[x_{k}\right]^{p(t)}-\left[x_{k}^{*}\right]^{p(t)}\right) \\
& \leq\left|\xi_{k-1}\right| \cdot \bar{p}\left(2^{\bar{p}-3}+1\right)\left(\left|\xi_{k}\right|^{p(t)}+\left|\xi_{k}\right|\left|x_{k}^{*}\right|^{p(t)-1}\right) \\
& \quad \leq \widetilde{r}_{k, 1}\left|\xi_{k-1}\right|^{p(t)+1}+\lambda_{k, 1}\left|\xi_{k}\right|^{p(t)+1}, \\
& \xi_{k}\left(\phi_{k}+\sum_{i=1}^{k-1} \beta_{i \sim(k-1)}\left(\left[x_{i+1}\right]^{p(t)}+\phi_{i}\right)\right) \\
& \leq\left|\xi_{k}\right|\left(\bar{\beta}_{1}\left|\xi_{1}\right|^{p(t)}+\bar{\beta}_{2}\left|x_{2}\right|^{p(t)}+\cdots+\bar{\beta}_{k}\left|x_{k}\right|^{p(t)}\right) \\
& \quad \leq \sum_{i=1}^{k-1} \bar{r}_{k, i}\left|\xi_{i}\right|^{p(t)+1}+\lambda_{k, 2}\left|\xi_{k}\right|^{p(t)+1},
\end{aligned}
$$

where $\bar{\beta}_{i}=\beta_{(i-1) \sim(k-1)}+\widetilde{c}\left(1+\sum_{j=i}^{k-1} \beta_{j \sim(k-1)}\right), \widetilde{r}_{k, 1}, \bar{r}_{k, 1}, \ldots, \bar{r}_{k, k-1}$ are positive constants, and $\lambda_{k, 1}$ and $\lambda_{k, 2}$ are positive constants 
dependent on $\widetilde{r}_{k, 1}$ and $\bar{r}_{k, 1}, \ldots, \bar{r}_{k, k-1}$, respectively. Substituting (15) and (16) into (14) leads to

$$
\begin{aligned}
\dot{V}_{k} \leq & -\sum_{i=1}^{k-1}\left(r_{i, i}-\widetilde{r}_{i+1,1}-\sum_{j=i+1}^{k} \bar{r}_{j, i}\right)\left|\xi_{i}\right|^{p(t)+1} \\
& +\xi_{k}\left(\left[x_{k+1}\right]^{p(t)}-\left[x_{k+1}^{*}\right]^{p(t)}\right)+\xi_{k}\left[x_{k+1}^{*}\right]^{p(t)} \\
& +\left(\lambda_{k, 1}+\lambda_{k, 2}\right)\left|\xi_{k}\right|^{p(t)+1} .
\end{aligned}
$$

Choose the virtual controller $x_{k+1}^{*}=-\beta_{k} \xi_{k}$ with $\beta_{k}=\left(r_{k, k}+\right.$ $\left.\lambda_{k, 1}+\lambda_{k, 2}\right)^{1 / \underline{p}}$, where $r_{k, k} \geq 1$ is a constant to be designed. Due to $\left(r_{k, k}+\lambda_{k, 1}+\lambda_{k, 2}\right)^{p(t) / \underline{p}} \geq\left(r_{k, k}+\lambda_{k, 1}+\lambda_{k, 2}\right)$, (17) becomes

$$
\begin{aligned}
\dot{V}_{k} \leq & -\sum_{i=1}^{k-1}\left(r_{i, i}-\tilde{r}_{i+1,1}-\sum_{j=i+1}^{k} \bar{r}_{j, i}\right)\left|\xi_{i}\right|^{p(t)+1} \\
& -r_{k, k}\left|\xi_{k}\right|^{p(t)+1}+\xi_{k}\left(\left[x_{k+1}\right]^{p(t)}-\left[x_{k+1}^{*}\right]^{p(t)}\right) .
\end{aligned}
$$

This completes the inductive step.

Step $n$. The Lyapunov function $V_{n}(\xi)=V_{n-1}\left(\xi_{1}, \ldots, \xi_{n-1}\right)+$ $(1 / 2) \xi_{n}^{2}=\sum_{i=1}^{n}(1 / 2) \xi_{i}^{2}$ and a positive constant $\lambda$ give

$$
\begin{aligned}
\dot{V}_{n} \leq & -\sum_{i=1}^{n-1}\left(r_{i, i}-\widetilde{r}_{i+1,1}-\sum_{j=i+1}^{n} \bar{r}_{j, i}\right)\left|\xi_{i}\right|^{p(t)+1} \\
& +\lambda\left|\xi_{n}\right|^{p(t)+1}+\xi_{n}\left([u]^{p(t)}-\left[x_{n+1}^{*}\right]^{p(t)}\right) \\
& +\xi_{n}\left[x_{n+1}^{*}\right]^{p(t)},
\end{aligned}
$$

where $\xi_{i}$ is defined as in (11) for $i=1, \ldots, n$. Choose virtual controller $x_{n+1}^{*}=-\beta_{n} \xi_{n}$ with $\beta_{n}=\left(r_{n, n}+\lambda\right)^{1 / p}$, where $r_{n, n} \geq 1$ is a constant to be designed. Due to $\left(r_{n, n}+\lambda\right)^{p(t) / \underline{p}} \geq\left(r_{n, n}+\lambda\right)$, (19) becomes

$$
\begin{aligned}
\dot{V}_{n} \leq & -\sum_{i=1}^{n-1}\left(r_{i, i}-\widetilde{r}_{i+1,1}-\sum_{j=i+1}^{n} \bar{r}_{j, i}\right)\left|\xi_{i}\right|^{p(t)+1} \\
& -r_{n, n}\left|\xi_{n}\right|^{p(t)+1}+\xi_{n}\left([u]^{p(t)}-\left[x_{n+1}^{*}\right]^{p(t)}\right) .
\end{aligned}
$$

3.3. Output Feedback Controller Design of System (1). For system (1), since output function is unknown, the state $x_{1}$ is exactly unknown. We construct the output-driven nonlinear reduced-order observer

$$
\begin{aligned}
\dot{\vec{z}}_{i}=-l_{i}\left(\left[\widehat{x}_{i}\right]^{p(t)}+\widehat{\phi}_{i-1}\right), & \\
\hat{x}_{i} & =\widehat{z}_{i}+l_{i} \widehat{x}_{i-1}, i=2, \ldots, n,
\end{aligned}
$$

where $\widehat{x}_{1}=y, \widehat{\phi}_{i-1}:=\phi_{i-1}\left(t, \widehat{x}_{1}, \ldots, \widehat{x}_{i-1}\right), i=2, \ldots, n$, and the observer gains $l_{i} \geq 1, i=2, \ldots, n$, are constants to be determined.

Remark 10. Compared with the observer in [29], since the unknown output function $h(\cdot)$ causes $x_{1}$ to be exactly unknown, a new output-driven nonlinear reduced-order observer (21) is designed without using $x_{1}$ but $y$ to rebuild unmeasured states. Moreover, not all but the first $n-1$ nonlinearities are used in the design of observer. For a second-order system Example 4.1 in [29], we can design an observer without using nonlinearity; see the simulation example in this paper for the details.

Based on $x_{n+1}^{*}=-\beta_{n} \xi_{n}=-\sum_{i=1}^{n} \beta_{i \sim n} x_{i}$, an output feedback controller is designed as

$$
u=-\sum_{i=1}^{n} \beta_{i \sim n} \widehat{x}_{i}=-\beta_{1 \sim n} y-\sum_{i=2}^{n} \beta_{i \sim n} \widehat{x}_{i} .
$$

Define the error

$$
e_{i}=z_{i}-\widehat{z}_{i}, \quad z_{i}=x_{i}-l_{i} x_{i-1}, i=2, \ldots, n .
$$

By (1), (21), and (23), one has

$$
\begin{aligned}
\dot{e}_{i}= & -l_{i}\left(\left[x_{i}\right]^{p(t)}-\left[\widehat{x}_{i}\right]^{p(t)}\right)+\left[x_{i+1}\right]^{p(t)}+\phi_{i} \\
& -l_{i}\left(\phi_{i-1}-\widehat{\phi}_{i-1}\right), \quad i=2, \ldots, n-1, \\
\dot{e}_{n}= & -l_{n}\left(\left[x_{n}\right]^{p(t)}-\left[\widehat{x}_{n}\right]^{p(t)}\right)+[u]^{p(t)}+\phi_{n} \\
& -l_{n}\left(\phi_{n-1}-\widehat{\phi}_{n-1}\right) .
\end{aligned}
$$

For the Lyapunov function $U(e)=\sum_{i=2}^{n}(1 / 2) e_{i}^{2}$, it follows from (21), (23), and (24) that

$$
\begin{aligned}
\dot{U}= & -\sum_{i=2}^{n} l_{i} e_{i}\left(\left[z_{i}+l_{i} x_{i-1}\right]^{p(t)}-\left[\widehat{z}_{i}+l_{i} x_{i-1}\right]^{p(t)}\right) \\
& -\sum_{i=2}^{n} l_{i} e_{i}\left(\left[\widehat{z}_{i}+l_{i} x_{i-1}\right]^{p(t)}-\left[\widehat{z}_{i}+l_{i} \widehat{x}_{i-1}\right]^{p(t)}\right) \\
& +\sum_{i=2}^{n-1} e_{i}\left[x_{i+1}\right]^{p(t)}+e_{n}[u]^{p(t)}+\sum_{i=2}^{n} e_{i} \phi_{i} \\
& -\sum_{i=2}^{n} l_{i} e_{i}\left(\phi_{i-1}-\widehat{\phi}_{i-1}\right) .
\end{aligned}
$$

By (23), Lemma 5 , and the fact that $1 \leq p \leq p(t) \leq \bar{p}$, we obtain for $i=2, \ldots, n$,

$$
\begin{aligned}
& -l_{i} e_{i}\left(\left[z_{i}+l_{i} x_{i-1}\right]^{p(t)}-\left[\widehat{z}_{i}+l_{i} x_{i-1}\right]^{p(t)}\right) \\
& =-l_{i}\left(\left(z_{i}+l_{i} x_{i-1}\right)-\left(\widehat{z}_{i}+l_{i} x_{i-1}\right)\right) \\
& \cdot\left(\left[z_{i}+l_{i} x_{i-1}\right]^{p(t)}-\left[\widehat{z}_{i}+l_{i} x_{i-1}\right]^{p(t)}\right) \\
& \quad \leq-2^{1-\bar{p}} l_{i}\left|e_{i}\right|^{p(t)+1} .
\end{aligned}
$$

Next, we give the estimate of the others on the right-hand side of (25) by Propositions 11-14, whose proofs are included in the Appendix. 
Proposition 11. There is a positive constant $b_{1}$, and positive constants $c_{1, i}, i=2, \ldots, n$, dependent on $b_{1}$ such that

$$
\begin{aligned}
& \sum_{i=2}^{n-1} e_{i}\left[x_{i+1}\right]^{p(t)}+\sum_{i=2}^{n} e_{i} \phi_{i} \\
& \quad \leq b_{1} \sum_{i=1}^{n}\left|\xi_{i}\right|^{p(t)+1}+\sum_{i=2}^{n} c_{1, i}\left|e_{i}\right|^{p(t)+1} .
\end{aligned}
$$

Proposition 12. There is a positive constant $b_{2}$, a positive constant $\alpha_{n}$ dependent on $b_{2}$, and positive constants $\alpha_{i}, i=$ $1, \ldots, n-1$, dependent on $b_{2}, l_{i+1}, \ldots, l_{n}$ such that

$$
\begin{gathered}
-\sum_{i=2}^{n} l_{i} e_{i}\left(\left[\widehat{z}_{i}+l_{i} x_{i-1}\right]^{p(t)}-\left[\widehat{z}_{i}+l_{i} \widehat{x}_{i-1}\right]^{p(t)}\right) \\
\leq b_{2} \sum_{i=1}^{n}\left|\xi_{i}\right|^{p(t)+1}+\alpha_{n}\left|e_{n}\right|^{p(t)+1} \\
+\sum_{i=2}^{n-1} \alpha_{i}\left(l_{i+1}, \ldots, l_{n}\right)\left|e_{i}\right|^{p(t)+1} \\
\quad+\alpha_{1}\left(l_{2}, \ldots, l_{n}\right)\left|x_{1}-\widehat{x}_{1}\right|^{p(t)+1} .
\end{gathered}
$$

Proposition 13. There is a positive constant $b_{3}$, a positive constant $\gamma_{n}$ dependent on $b_{3}$, and positive constants $\gamma_{i}, i=$ $1, \ldots, n-1$, dependent on $b_{3}, l_{i+1}, \ldots, l_{n}$ such that

$$
\begin{aligned}
& -\sum_{i=2}^{n} l_{i} e_{i}\left(\phi_{i-1}-\widehat{\phi}_{i-1}\right) \\
& \leq b_{3} \sum_{i=1}^{n}\left|\xi_{i}\right|^{p(t)+1}+\gamma_{n}\left|e_{n}\right|^{p(t)+1} \\
& \quad+\sum_{i=2}^{n-1} \gamma_{i}\left(l_{i+1}, \ldots, l_{n}\right)\left|e_{i}\right|^{p(t)+1} \\
& \quad+\gamma_{1}\left(l_{2}, \ldots, l_{n}\right)\left|x_{1}-\widehat{x}_{1}\right|^{p(t)+1} .
\end{aligned}
$$

Proposition 14. There is a positive constant $b_{4}$, a positive constant $\sigma_{n}$ dependent on $b_{4}$, and positive constants $\sigma_{i}, i=$ $1, \ldots, n-1$, dependent on $l_{i+1}, \ldots, l_{n}$ such that

$$
\begin{aligned}
e_{n}[u]^{p(t)} \leq & b_{4}\left|\xi_{n}\right|^{p(t)+1}+\sigma_{n}\left|e_{n}\right|^{p(t)+1} \\
& +\sum_{i=2}^{n-1} \sigma_{i}\left(l_{i+1}, \ldots, l_{n}\right)\left|e_{i}\right|^{p(t)+1} \\
& +\sigma_{1}\left(l_{2}, \ldots, l_{n}\right)\left|x_{1}-\widehat{x}_{1}\right|^{p(t)+1}
\end{aligned}
$$

To estimate the term $\xi_{n}\left([u]^{p(t)}-\left[x_{n+1}^{*}\right]^{p(t)}\right)$ in (20), we give the following proposition whose proof is also included in the Appendix.
Proposition 15. There is a positive constant $b_{5}$, a positive constant $\omega_{n}$ dependent on $b_{5}$, and positive constants $\omega_{i}, i=$ $1, \ldots, n-1$, dependent on $b_{5}, l_{i+1}, \ldots, l_{n}$ such that

$$
\begin{aligned}
\xi_{n}\left([u]^{p(t)}-\left[x_{n+1}^{*}\right]^{p(t)}\right) \\
\leq b_{5}\left|\xi_{n}\right|^{p(t)+1}+\omega_{n}\left|e_{n}\right|^{p(t)+1} \\
\quad+\sum_{i=2}^{n-1} \omega_{i}\left(l_{i+1}, \ldots, l_{n}\right)\left|e_{i}\right|^{p(t)+1} \\
\quad+\omega_{1}\left(l_{2}, \ldots, l_{n}\right)\left|x_{1}-\widehat{x}_{1}\right|^{p(t)+1} .
\end{aligned}
$$

Define $T(\xi, e)=V_{n}(\xi)+U(e)=\sum_{i=1}^{n}(1 / 2) \xi_{i}^{2}+\sum_{i=2}^{n}(1 / 2) e_{i}^{2}$. By (20), (25), (26), and Propositions 11-15, one obtains

$$
\begin{aligned}
\dot{T} & \leq-\sum_{i=1}^{n-1}\left(r_{i, i}-\widetilde{r}_{i+1,1}-\sum_{j=i+1}^{n} \bar{r}_{j, i}-\left(b_{1}+b_{2}+b_{3}\right)\right) \\
& \cdot\left|\xi_{i}\right|^{p(t)+1}-\left(r_{n, n}-\left(b_{1}+b_{2}+b_{3}+b_{4}+b_{5}\right)\right) \\
& \cdot\left|\xi_{n}\right|^{p(t)+1}-\left(2^{1-\bar{p}} l_{n}-\theta_{n}\right)\left|e_{n}\right|^{p(t)+1} \\
& -\sum_{i=2}^{n-1}\left(2^{1-\bar{p}} l_{i}-\theta_{i}\left(l_{i+1}, \ldots, l_{n}\right)\right)\left|e_{i}\right|^{p(t)+1} \\
& +\theta_{1}\left(l_{2}, \ldots, l_{n}\right)\left|x_{1}-\widehat{x}_{1}\right|^{p(t)+1}
\end{aligned}
$$

where $\theta_{i}=\alpha_{i}+\gamma_{i}+\sigma_{i}+\omega_{i}+c_{1, i}$ with $c_{1,1}=0, i=1, \ldots, n$, it is easy to see that $\theta_{n}$ is a positive constant, and $\theta_{i}, i=1, \ldots, n-$ 1 , are positive constants dependent on $l_{i+1}, \ldots, l_{n}$. For some positive constants $b_{0}, \widetilde{b}_{1}, \ldots, \widetilde{b}_{n}$, design $r_{1,1}, \ldots, r_{n, n}$ such that

$$
\begin{aligned}
& r_{1,1} \geq \max \left\{\widetilde{r}_{2,1}+\sum_{j=2}^{n} \bar{r}_{j, 1}+b_{1}+b_{2}+b_{3}+b_{0}+\widetilde{b}_{1}, 1\right\}, \\
& r_{i, i} \geq \max \left\{\tilde{r}_{i+1,1}+\sum_{j=i+1}^{n} \bar{r}_{j, i}+b_{1}+b_{2}+b_{3}+\tilde{b}_{i}, 1\right\}, \\
& i=2, \ldots, n-1, \\
& r_{n, n} \geq \max \left\{b_{1}+b_{2}+b_{3}+b_{4}+b_{5}+\tilde{b}_{n}, 1\right\},
\end{aligned}
$$

and for some positive constants $\widetilde{d}_{2}, \ldots, \widetilde{d}_{n}$, choose $l_{2}, \ldots, l_{n}$ as

$$
\begin{aligned}
& l_{n} \geq \max \left\{2^{\bar{p}^{-1}}\left(\tilde{d}_{n}+\theta_{n}\right), 1\right\}, \\
& l_{i} \geq \max \left\{2^{\bar{p}^{-1}}\left(\tilde{d}_{i}+\theta_{i}\left(l_{i+1}, \ldots, l_{n}\right)\right), 1\right\},
\end{aligned}
$$

$$
i=n-1, \ldots, 2 \text {. }
$$


By (33) and (34), (32) becomes

$$
\begin{aligned}
\dot{T} \leq & -b_{0}\left|\xi_{1}\right|^{p(t)+1}-\sum_{i=1}^{n} \widetilde{b}_{i}\left|\xi_{i}\right|^{p(t)+1}-\sum_{i=2}^{n} \widetilde{d}_{i}\left|e_{i}\right|^{p(t)+1} \\
& +\theta_{1}\left(l_{2}, \ldots, l_{n}\right)\left|x_{1}-\widehat{x}_{1}\right|^{p(t)+1} .
\end{aligned}
$$

\subsection{Stability and Convergence Analysis}

Theorem 16. If Assumption 8 holds for system (1), there is a maximal open sector $\Omega=(1-\bar{\rho}, 1+\bar{\rho})$ with $\bar{\rho}$ being a positive constant, as long as the unknown output function $h(\cdot)$ belongs to any closed sector included in $\Omega$, under the output feedback controller (21) and (22):

(i) the solutions of the closed-loop system (1), (21), (22) are well-defined on $[0,+\infty)$,

(ii) the equilibrium point $x=0$ is globally uniformly asymptotically stable.

Proof. (i) We firstly give the choice of $r_{1,1}, \ldots, r_{n, n}, \beta_{1}, \ldots, \beta_{n}$. At Step 1 , for any given $\widetilde{r}_{2,1}, \bar{r}_{2,1}, \ldots, \bar{r}_{n, 1}, b_{1}, b_{2}, b_{3}, b_{0}, \tilde{b}_{1}$, by (33), we can choose $r_{1,1}$; then $\beta_{1}$ can be calculated. At step 2 , for any given $\widetilde{r}_{3,1}, \bar{r}_{3,2}, \ldots, \bar{r}_{n, 2}, b_{1}, b_{2}, b_{3}, \tilde{b}_{2}$, by (33), we can choose $r_{2,2}$; then $\beta_{2}$ can be calculated when $\lambda_{2,1}, \lambda_{2,2}$ have been obtained by (15) and (16). One by one, at Step n, for any given $b_{1}, b_{2}, b_{3}, b_{4}, b_{5}, \widetilde{b}_{n}$, by (33), we can choose $r_{n, n}$; then $\beta_{n}$ can be calculated.

Secondly, we give the choice of $l_{n}, \ldots, l_{2}$. For the given $b_{1}, b_{2}, b_{3}, b_{4}, b_{5}$, we can get $c_{1, n}, \alpha_{n}, \gamma_{n}, \sigma_{n}, \omega_{n}$. Then for any given $\widetilde{d}_{n}$, by (34), we can choose $l_{n}$. Next, for the given $b_{1}, b_{2}, b_{3}, b_{5}$, one get $c_{1, n-1}, \alpha_{n-1}\left(l_{n}\right), \gamma_{n-1}\left(l_{n}\right), \sigma_{n-1}\left(l_{n}\right), \omega_{n-1}\left(l_{n}\right)$. Then for any given $\widetilde{d}_{n-1}, l_{n-1}$ can be chosen by (34). In turn, we can get $l_{n-2}, \ldots, l_{2}$.

Finally, we give the sector of output function. Denote $\kappa=$ $\left(\widetilde{r}_{2,1}, \ldots, \widetilde{r}_{n, 1}, \bar{r}_{2,1}, \ldots, \bar{r}_{n, 1}, \ldots, \bar{r}_{n, n-2}, \bar{r}_{n, n-1}, b_{1}, \ldots, b_{5}, b_{0}, \widetilde{b}_{1}\right.$, $\left.\ldots, \tilde{b}_{n}, \tilde{d}_{2}, \ldots, \widetilde{d}_{n}\right), \Omega_{l}=\left\{l=\left(l_{2}, \ldots, l_{n}\right) \mid r_{1,1} \geq\right.$ $\max \left\{\tilde{r}_{2,1}+\sum_{j=2}^{n} \bar{r}_{j, 1}+b_{1}+b_{2}+b_{3}+b_{0}+\tilde{b}_{1}, 1\right\}, r_{i, i} \geq$ $\max \left\{\widetilde{r}_{i+1,1}+\sum_{j=i+1}^{n} \bar{r}_{j, i}+b_{1}+b_{2}+b_{3}+\tilde{b}_{i}, 1\right\}, i=2, \ldots, n-1, r_{n, n} \geq$ $\max \left\{b_{1}+b_{2}+b_{3}+b_{4}+b_{5}+\tilde{b}_{n}, 1\right\}, l_{n} \geq \max \left\{2^{\bar{p}^{-1}}\left(\tilde{d}_{n}+\theta_{n}\right), 1\right\}, l_{i} \geq$ $\left.\max \left\{2^{\bar{p}-1}\left(\widetilde{d}_{i}+\theta_{i}\left(l_{i+1}, \ldots, l_{n}\right)\right), 1\right\}, i=n-1, \ldots, 2\right\}$, and

$$
\begin{aligned}
& \rho=\rho\left(l_{2}, \ldots, l_{n}\right)=\min \left\{\left(\frac{b_{0}}{2 \theta_{1}\left(l_{2}, \ldots, l_{n}\right)}\right)^{1 /(\bar{p}+1)},\right. \\
& \left.\left(\frac{b_{0}}{2 \theta_{1}\left(l_{2}, \ldots, l_{n}\right)}\right)^{1 /(\underline{p}+1)}\right\} .
\end{aligned}
$$

In fact, one can determine the supremum of $\rho$; that is, select constant

$$
\bar{\rho}=\sup _{\kappa \in R^{+}, l \in \Omega_{l}} \rho\left(l_{2}, \ldots, l_{n}\right)
$$

as the supremum of $\rho$. Hence, the maximal open sector of output function is $\Omega=(1-\bar{\rho}, 1+\bar{\rho})$. When output function $h\left(x_{1}\right)$ in (1) belongs to any closed sector $[1-\bar{\rho}+\epsilon, 1+\bar{\rho}-\epsilon] \subset \Omega$ with $0<\epsilon<\bar{\rho}$, by Definition 1 ,

$$
\begin{aligned}
& \left(h\left(x_{1}\right)-(1-\bar{\rho}+\epsilon) x_{1}\right)\left(h\left(x_{1}\right)-(1+\bar{\rho}-\epsilon) x_{1}\right) \\
& \quad \leq 0 \Longrightarrow \\
& \left(h\left(x_{1}\right)-(1+\bar{\rho}-\epsilon) x_{1}\right) x_{1} \leq 0 \\
& \quad \leq\left(h\left(x_{1}\right)-(1-\bar{\rho}+\epsilon) x_{1}\right) x_{1} \Longrightarrow \\
& (1-\bar{\rho}+\epsilon) x_{1}^{2} \leq h\left(x_{1}\right) x_{1} \leq(1+\bar{\rho}-\epsilon) x_{1}^{2} \Longrightarrow \\
& -(\bar{\rho}-\epsilon) x_{1}^{2} \leq\left(h\left(x_{1}\right)-x_{1}\right) x_{1} \leq(\bar{\rho}-\epsilon) x_{1}^{2} \Longrightarrow \\
& \left|x_{1}-h\left(x_{1}\right)\right| \leq(\bar{\rho}-\epsilon)\left|x_{1}\right|,
\end{aligned}
$$

from which one has

$$
\begin{aligned}
\left|x_{1}-\widehat{x}_{1}\right|^{p(t)+1} & =\left|\xi_{1}-h\left(\xi_{1}\right)\right|^{p(t)+1} \\
& \leq(\bar{\rho}-\epsilon)^{p(t)+1}\left|\xi_{1}\right|^{p(t)+1} .
\end{aligned}
$$

With the help of $p+1 \leq p(t)+1 \leq \bar{p}+1$, (36), and (37), we derive

$$
(\bar{\rho}-\epsilon)^{p(t)+1}<\rho^{p(t)+1} \leq \rho^{\bar{p}+1}+\rho^{p^{+1}} \leq \frac{b_{0}}{\theta_{1}\left(l_{2}, \ldots, l_{n}\right)} .
$$

From (39) and (40), (35) becomes

$$
\dot{T} \leq-\sum_{i=1}^{n} \widetilde{b}_{i}\left|\xi_{i}\right|^{p(t)+1}-\sum_{i=2}^{n} \widetilde{d}_{i}\left|e_{i}\right|^{p(t)+1}
$$

Motivated by [29], with the help of $\underline{p}+p(t)+1 \leq \underline{p}+\bar{p}+1 \leq$ $p(t)+1+\bar{p}$, one obtains

$$
\begin{aligned}
& \left|\xi_{i}\right|^{p+\bar{p}+1} \leq\left|\xi_{i}\right|^{p(t)+1}\left(\left|\xi_{i}\right|^{p}+\left|\xi_{i}\right|^{\bar{p}}\right) \Longrightarrow \\
& \left|\xi_{i}\right|^{p(t)+1} \geq \frac{\left|\xi_{i}\right|^{\bar{p}+1}}{1+\left|\xi_{i}\right|^{\bar{p}-\underline{p}}}, \\
& i=1, \ldots, n \text {, } \\
& \left|e_{i}\right|^{\underline{p}+\bar{p}+1} \leq\left|e_{i}\right|^{p(t)+1}\left(\left|e_{i}\right|^{\mid \underline{p}}+\left|e_{i}\right|^{\bar{p}}\right) \Longrightarrow \\
& \left|e_{i}\right|^{p(t)+1} \geq \frac{\left|e_{i}\right|^{\bar{p}+1}}{1+\left|\xi_{i}\right|^{\bar{p}-\underline{p}}},
\end{aligned}
$$

from which (41) becomes

$$
\dot{T} \leq-\left(\sum_{i=1}^{n} \frac{\tilde{b}_{i}\left|\xi_{i}\right|^{\bar{p}+1}}{1+\left|\xi_{i}\right|^{\bar{p}-\underline{p}}}+\sum_{i=2}^{n} \frac{\widetilde{d}_{i}\left|e_{i}\right|^{\bar{p}+1}}{1+\left|e_{i}\right|^{\bar{p}-\underline{p}}}\right)=:-W(\xi, e) .
$$

It is easy to see that $W(\xi, e)$ is a continuous and positive function with respect to $(\xi, e)$. 

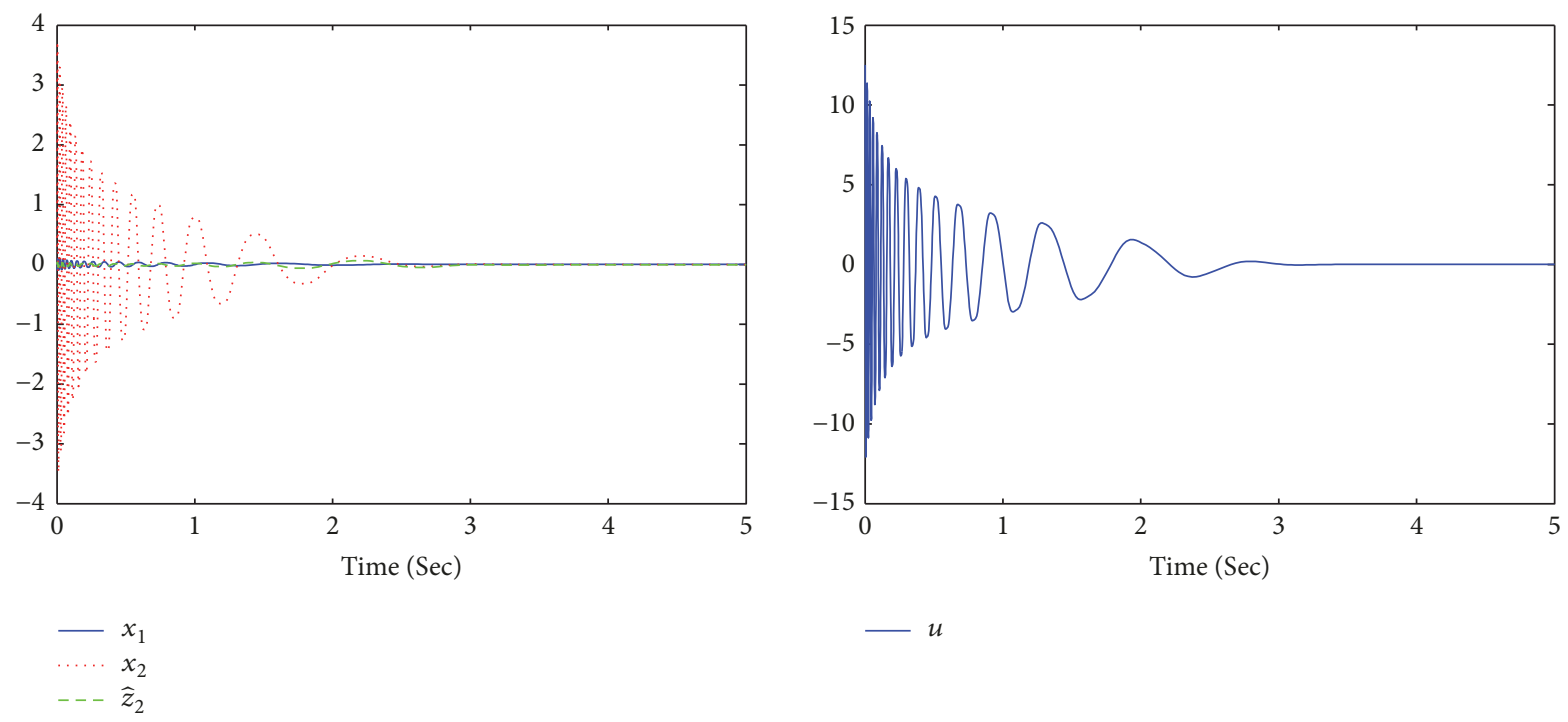

FIGURE 1: The responses of the closed-loop system (46), (47) with $h\left(x_{1}\right)=x_{1}$.

By the transformations (11), system (1) can be transformed into a $\xi$-system:

$$
\dot{\xi}_{i}(t)=f_{i}(t, \xi(t), u(t)), \quad i=1, \ldots, n,
$$

where $f_{i}(\cdot)$ is a continuous function with $f_{i}(t, 0)=0$. Denote $\omega=\left(\xi_{1}, \ldots, \xi_{n}, e_{2}, \ldots, e_{n}\right)^{\top}$, by the existence and continuation of the solution, the solution $\omega(t)$ of $\omega$-system (24), (44) is defined on $\left[0, t_{m}\right)$ with $0<t_{m} \leq+\infty$.

Due to $T(\omega)=\sum_{i=1}^{n}(1 / 2) \xi_{i}^{2}+\sum_{i=2}^{n}(1 / 2) e_{i}^{2}$, it is obvious that $T(\omega)$ is a continuously differentiable, positive definite, and radially unbounded function. By Lemma 6 , there are class $\mathscr{K}_{\infty}$ functions $\pi_{1}(\cdot)$ and $\pi_{2}(\cdot)$ such that

$$
\pi_{1}(\|\omega\|) \leq T(\omega) \leq \pi_{2}(\|\omega\|), \quad \forall \omega \in R^{2 n-1}
$$

Since $\pi_{1}(\cdot)$ is a class $\mathscr{K}_{\infty}$ function, then for any $\varepsilon>0$, one can always find a $\delta=\delta(\varepsilon)$ with $\delta>\varepsilon>0$ such that $\pi_{2}(\varepsilon) \leq$ $\pi_{1}(\delta)$. If $\|\omega(0)\|<\varepsilon$, by (43) and (45), $\pi_{1}(\|\omega(t)\|) \leq T(\omega(t)) \leq$ $T(\omega(0)) \leq \pi_{2}(\|\omega(0)\|) \leq \pi_{2}(\varepsilon) \leq \pi_{1}(\delta)$ for all $t \in\left[0, t_{m}\right)$, which means that $\|\omega(t)\| \leq \delta$ for all $t \in\left[0, t_{m}\right)$. Hence, $t_{m}$ is not an escape time; that is, $\omega(t)$ is well-defined on $[0,+\infty)$, so is $x(t)$.

(ii) Since $t_{m}=+\infty$, from (43), (45), and Lemma 7, we know that the equilibrium point $\omega=0$ of $\omega$-system (24), (44) is globally uniformly asymptotically stable. By the continuity of $x_{i}^{*}\left(\xi_{i-1}\right)$ on $\xi_{i-1}$ and $x_{i}^{*}(0)=0$, it is easy to recursively prove that the equilibrium point $x=0$ of the closed-loop system (1), (21), (22) is globally uniformly asymptotically stable.

Remark 17. Since the power of system is time-varying, a new function (36) is given to acquire its supremum, which is used to determine the maximal open sector of output function. Moreover, a rigorous analysis is given to establish the stability of the closed-loop system.

\section{Simulation Example}

Consider the second-order nonlinear system in [29]

$$
\begin{aligned}
& \dot{x}_{1}=\left[x_{2}\right]^{2+\cos (t)} \\
& \dot{x}_{2}=[u]^{2+\cos (t)}+x_{1}^{1 / 3}\left|x_{2}\right|^{\cos (t)+5 / 3}, \\
& y=h\left(x_{1}\right) .
\end{aligned}
$$

As discussed in [29], Assumption 8 holds and $p=1, \bar{p}=$ 3. Following the design process as in Section 3 in this paper, we can design output feedback controller without using the nonlinearity $x_{1}^{1 / 3}\left|x_{2}\right|^{\cos (t)+5 / 3}$,

$$
\begin{aligned}
\dot{\vec{z}}_{2} & =-l_{2}\left[\widehat{x}_{2}\right]^{p(t)}, \quad \widehat{x}_{2}=\widehat{z}_{2}+l_{2} y, \\
u & =-\beta_{1} \beta_{2} y-\beta_{2} \widehat{x}_{2} .
\end{aligned}
$$

Choose $\kappa=\left(\widetilde{r}_{2,1}, \bar{r}_{2,1}, b_{1}, b_{2}, b_{4}, b_{5}, b_{0}, \tilde{b}_{1}, \tilde{b}_{2}, \tilde{d}_{2}\right)=$ $(0.1,0.1,0.1,0.1,0.1,0.1,0.4,0.1,0.1,0.4)$. By choosing $r_{1,1}=1, r_{2,2}=1$, we obtain $\beta_{1}=1, \beta_{2}=9.63$, and choose $l=l_{2}=12$.

When $h\left(x_{1}\right)=x_{1}$ as in [29], it means that we do not need to give the sector of the output function. In simulation, under the output feedback controller (47), by selecting the initial conditions $x_{1}(0)=-0.1, x_{2}(0)=0.3, \widehat{z}_{2}(0)=0$, Figure 1 demonstrates the effectiveness of control scheme.

When $h\left(x_{1}\right) \neq x_{1}$, the design method in [29] is inapplicable. Following the design process as in Section 3 in this paper, we can get a sector of output function, that is, $[1-\rho, 1+\rho]$ with $\rho=\rho\left(l_{2}\right)=\min \left\{\left(b_{0} / 2 \theta_{1}\left(l_{2}\right)\right)^{1 /(\bar{p}+1)},\left(b_{0} / 2 \theta_{1}\left(l_{2}\right)\right)^{1 /(\underline{p}+1)}\right\}=$ 0.0131. In simulation, we choose $h\left(x_{1}\right)=x_{1}+0.01\left|x_{1}\right|$ which is only continuous but not differentiable; $h\left(x_{1}\right)$ belongs to sector $[0.9869,1.0131]$ obviously. By selecting the initial conditions $x_{1}(0)=0.1, x_{2}(0)=-0.3, \widehat{z}_{2}(0)=0.01$, Figure 2 illustrates the validness of control scheme. 

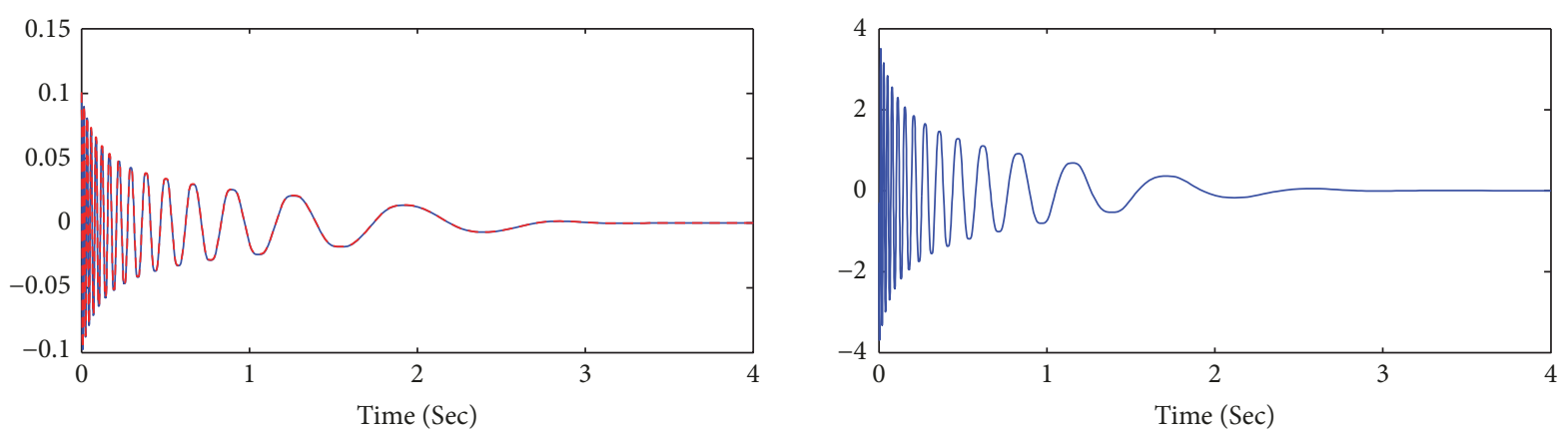

$x_{1}$

$---y$

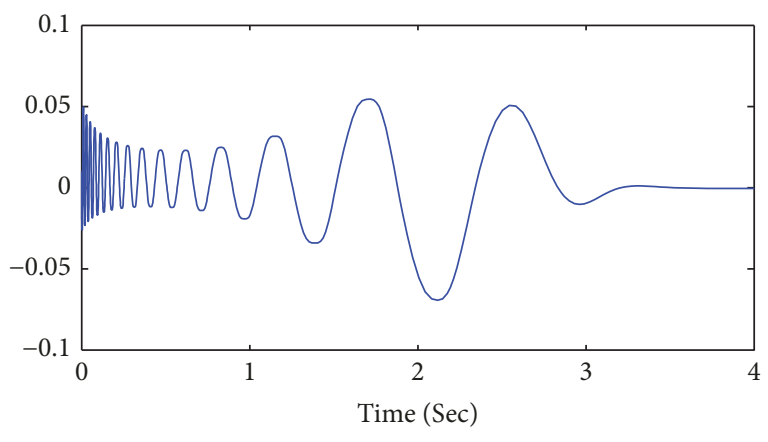

$-x_{2}$

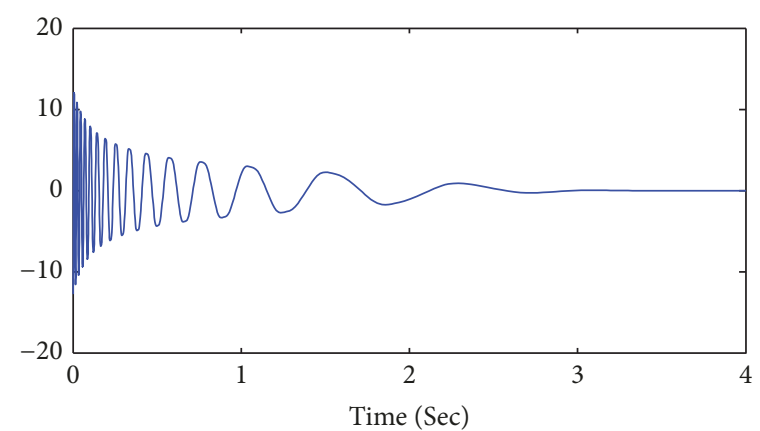

$-\widehat{z}_{2}$

$-u$

FIGURE 2: The responses of the closed-loop system (46), (47) with $h\left(x_{1}\right)=x_{1}+0.01\left|x_{1}\right|$.

\section{Conclusions}

In this paper, output feedback stabilization of nonlinear systems with a time-varying power and unknown continuous output function is studied. By constructing a new nonlinear reduced-order observer together with adding a power integrator method, a new function to determine the maximal open sector $\Omega$ of output function is given. As long as output function belongs to any closed sector included in $\Omega$, an output feedback controller is constructed to guarantee that the equilibrium point of the closed-loop system is globally uniformly asymptotically stable.

For the future work, an interesting problem is for more general nonlinear systems with time-varying powers, that is, nonlinear systems with different time-varying powers or under the weaker condition on nonlinearities, can we design a stable output feedback controller?

\section{Appendix}

Proof of Proposition 11. By (8), (11), and Lemmas 2 and 3, we obtain

$$
\begin{gathered}
\sum_{i=2}^{n-1} e_{i}\left[x_{i+1}\right]^{p(t)}+\sum_{i=2}^{n} e_{i} \phi_{i} \\
\leq \sum_{i=2}^{n-1}\left|e_{i}\right|\left|x_{i+1}\right|^{p(t)}
\end{gathered}
$$

$$
\begin{aligned}
& +\sum_{i=2}^{n} \widetilde{c}\left|e_{i}\right|\left(\left|x_{1}\right|^{p(t)}+\cdots+\left|x_{i}\right|^{p(t)}\right) \\
\leq & b_{1} \sum_{i=1}^{n}\left|\xi_{i}\right|^{p(t)+1}+\sum_{i=2}^{n} c_{1, i}\left|e_{i}\right|^{p(t)+1},
\end{aligned}
$$

where $b_{1}>0$ is a constant, and $c_{1, i}>0, i=2, \ldots, n$, are constants dependent on $b_{1}$.

Proof of Proposition 12 . By (11), (21), and (23), one has for $i=2, \ldots, n$,

$$
\begin{aligned}
x_{i}-\widehat{x}_{i} & =e_{i}+l_{i}\left(x_{i-1}-\widehat{x}_{i-1}\right) \\
& =e_{i}+\sum_{j=2}^{i-1} l_{(j+1) \sim i} e_{j}+l_{2 \sim i}\left(x_{1}-\widehat{x}_{1}\right), \\
\widehat{z}_{i}+l_{i} x_{i-1} & =x_{i}-e_{i}=\xi_{i}-\beta_{i-1} \xi_{i-1}-e_{i},
\end{aligned}
$$

from which, by Lemmas $2-4$, we derive

$$
\begin{aligned}
& -l_{i} e_{i}\left(\left[\widehat{z}_{i}+l_{i} x_{i-1}\right]^{p(t)}-\left[\widehat{z}_{i}+l_{i} \widehat{x}_{i-1}\right]^{p(t)}\right) \leq l_{i}\left|e_{i}\right| \\
& \cdot \bar{p}\left(2^{\bar{p}-3}+1\right)\left(\left|l_{i}\left(x_{i-1}-\widehat{x}_{i-1}\right)\right|^{p(t)}\right. \\
& \left.\quad+\left|l_{i}\left(x_{i-1}-\widehat{x}_{i-1}\right)\right|\left|\widehat{z}_{i}+l_{i} x_{i-1}\right|^{p(t)-1}\right) \leq l_{i}\left|e_{i}\right| \\
& \cdot \bar{p}\left(2^{\bar{p}-3}+1\right)\left(\mid l_{i} e_{i-1}+l_{(i-1) \sim i} e_{i-2}+\cdots+l_{3 \sim i} e_{2}\right.
\end{aligned}
$$




$$
\begin{aligned}
& +\left.l_{2 \sim i}\left(x_{1}-\widehat{x}_{1}\right)\right|^{p(t)}+\mid l_{i} e_{i-1}+l_{(i-1) \sim i} e_{i-2}+\cdots \\
& \left.+l_{3 \sim i} e_{2}+l_{2 \sim i}\left(x_{1}-\widehat{x}_{1}\right)|| \xi_{i}-\beta_{i-1} \xi_{i-1}-\left.e_{i}\right|^{p(t)-1}\right) \\
& \leq \frac{b_{2}}{2}\left(\left|\xi_{i-1}\right|^{p(t)+1}+\left|\xi_{i}\right|^{p(t)+1}\right)+c_{2, i}\left|e_{i}\right|^{p(t)+1} \\
& +\sum_{j=2}^{i-1} \alpha_{i, j}\left(l_{j+1}, \ldots, l_{i}\right)\left|e_{j}\right|^{p(t)+1}+\alpha_{i, 1}\left(l_{2}, \ldots, l_{i}\right) \mid x_{1} \\
& -\left.\widehat{x}_{1}\right|^{p(t)+1}
\end{aligned}
$$

where $e_{1}=0, b_{2}>0$ is a constant, $c_{2, i}>0$ is a constant dependent on $b_{2}$, and $\alpha_{i, j}>0, j=1, \ldots, i-1$, are constants dependent on $b_{2}, l_{j+1}, \ldots, l_{i}$. From (A.4), it follows that

$$
\begin{aligned}
& -\sum_{i=2}^{n} l_{i} e_{i}\left(\left[\widehat{z}_{i}+l_{i} x_{i-1}\right]^{p(t)}\right. \\
& \quad \leq b_{2} \sum_{i=1}^{n}\left|\xi_{i}\right|^{p(t)+1}+\alpha_{n}\left|e_{n}\right|^{p(t)+1}
\end{aligned}
$$

$$
\begin{aligned}
& +\sum_{j=2}^{n-1} \alpha_{j}\left(l_{j+1}, \ldots, l_{n}\right)\left|e_{j}\right|^{p(t)+1} \\
& +\alpha_{1}\left(l_{2}, \ldots, l_{n}\right)\left|x_{1}-\widehat{x}_{1}\right|^{p(t)+1},
\end{aligned}
$$

where $\alpha_{j}=\sum_{i=j+1}^{n} \alpha_{i, j}\left(l_{j+1}, \ldots, l_{i}\right)+c_{2, j}$ with $c_{2,1}=0, j=$ $1, \ldots, n$, that is, $\alpha_{n}>0$ is a constant dependent on $b_{2}$, and $\alpha_{j}>$ $0, j=1, \ldots, n-1$, are constants dependent on $b_{2}, l_{j+1}, \ldots, l_{n}$.

Proof of Proposition 13. By (A.2), Assumption 8, and Lemmas 2 and 3 , one has

$$
\begin{aligned}
& \left|\phi_{1}\left(t, x_{1}\right)-\phi_{1}\left(t, \hat{x}_{1}\right)\right| \leq c \sum_{j=1}^{N}\left(\left|x_{1}-\widehat{x}_{1}\right|^{a_{1, j}(t)}\right. \\
& \left.\cdot\left(\left|x_{1}\right|^{b_{1, j}(t)}+\left|x_{1}-\left(x_{1}-\widehat{x}_{1}\right)\right|^{b_{1, j}(t)}\right)\right) \\
& \leq \widehat{c} \sum_{j=1}^{N}\left(\left|x_{1}-\widehat{x}_{1}\right|^{a_{1, j}(t)}\left|x_{1}\right|^{b_{1, j}(t)}+\left|x_{1}-\widehat{x}_{1}\right|^{p(t)}\right),
\end{aligned}
$$

and for $i=2, \ldots, n$,

$$
\begin{aligned}
& \left|\phi_{i}\left(t, x_{1}, \ldots, x_{i}\right)-\phi_{i}\left(t, \widehat{x}_{1}, \ldots, \widehat{x}_{i}\right)\right| \leq c \sum_{j=1}^{N}\left(\sum_{k=1}^{i}\left(\left|x_{k}-\widehat{x}_{k}\right|^{a_{k, j}(t)} \sum_{q=1}^{i}\left(\left|x_{q}\right|^{b_{k, j}(t)}+\left|\widehat{x}_{q}\right|^{b_{k, j}(t)}\right)\right)\right) \leq c \sum_{j=1}^{N}\left(\left|x_{1}-\widehat{x}_{1}\right|^{a_{1, j}(t)}\right. \\
& \cdot\left(\left|x_{1}\right|^{b_{1, j}(t)}+\left|x_{1}-\left(x_{1}-\widehat{x}_{1}\right)\right|^{b_{1, j}(t)}\right)+\left|x_{1}-\widehat{x}_{1}\right|^{a_{1, j}(t)} \sum_{q=2}^{i}\left(\left|x_{q}\right|^{b_{1, j}(t)}\right. \\
& \left.+\left|x_{q}-\left(e_{q}+l_{q} e_{q-1}+l_{(q-1) \sim q} e_{q-2}+\cdots+l_{3 \sim q} e_{2}+l_{2 \sim q}\left(x_{1}-\widehat{x}_{1}\right)\right)\right|^{b_{1, j}(t)}\right) \\
& +\sum_{k=2}^{i}\left(\left|e_{k}+l_{k} e_{k-1}+l_{(k-1) \sim k} e_{k-2}+\cdots+l_{3 \sim k} e_{2}+l_{2 \sim k}\left(x_{1}-\widehat{x}_{1}\right)\right|^{a_{k, j}(t)}\left(\left|x_{1}\right|^{b_{k, j}(t)}+\left|x_{1}-\left(x_{1}-\widehat{x}_{1}\right)\right|^{b_{k, j}(t)}\right)\right. \\
& +\left|e_{k}+l_{k} e_{k-1}+l_{(k-1) \sim k} e_{k-2}+\cdots+l_{3 \sim k} e_{2}+l_{2 \sim k}\left(x_{1}-\widehat{x}_{1}\right)\right|^{a_{k, j}(t)} \\
& \left.\left.\cdot \sum_{q=2}^{i}\left(\left|x_{q}\right|^{b_{k, j}(t)}+\left|x_{q}-\left(e_{q}+l_{q} e_{q-1}+l_{(q-1) \sim q} e_{q-2}+\cdots+l_{3 \sim q} e_{2}+l_{2 \sim q}\left(x_{1}-\widehat{x}_{1}\right)\right)\right|^{b_{k, j}(t)}\right)\right)\right) \leq \widehat{c} \sum_{j=1}^{N}\left(\left|x_{1}-\widehat{x}_{1}\right|^{a_{1, j}(t)}\right. \\
& \cdot \sum_{q=1}^{i}\left|x_{q}\right|^{b_{1, j}(t)}+\bar{\gamma}_{i, i}\left|e_{i}\right|^{p(t)}+\sum_{k=2}^{i-1} \bar{\gamma}_{i, k}\left(l_{k+1}, \ldots, l_{i}\right)\left|e_{k}\right|^{p(t)}+\bar{\gamma}_{i, 1}\left(l_{2}, \ldots, l_{i}\right)\left|x_{1}-\widehat{x}_{1}\right|^{p(t)} \\
& \left.+\sum_{k=2}^{i}\left(\left|e_{k}+l_{k} e_{k-1}+l_{(k-1) \sim k} e_{k-2}+\cdots+l_{3 \sim k} e_{2}+l_{2 \sim k}\left(x_{1}-\widehat{x}_{1}\right)\right|^{a_{k, j}(t)} \sum_{q=1}^{i}\left|x_{q}\right|^{b_{k, j}(t)}\right)\right)
\end{aligned}
$$


where $\widehat{c}>0$ is a constant, $\bar{\gamma}_{i, i}$ is a positive constant, and $\bar{\gamma}_{i, k}, k=1, \ldots, i-1$, are positive constants dependent on $l_{k+1}, \ldots, l_{i}$. From (11), (A.6), (A.7), and Lemmas 2 and 3, it follows that for $i=2, \ldots, n$,

$$
\begin{aligned}
& -l_{i} e_{i}\left(\phi_{i-1}-\widehat{\phi}_{i-1}\right) \\
& \leq l_{i}\left|e_{i}\right|\left|\phi_{i-1}\left(t, x_{1}, \ldots, x_{i-1}\right)-\phi_{i-1}\left(t, \widehat{x}_{1}, \ldots, \widehat{x}_{i-1}\right)\right| \\
& \leq \frac{b_{3}}{n} \sum_{j=1}^{i-1}\left|\xi_{j}\right|^{p(t)+1}+c_{3, i}\left|e_{i}\right|^{p(t)+1} \\
& \quad+\sum_{j=2}^{i-1} \gamma_{i, j}\left(l_{j+1}, \ldots, l_{i}\right)\left|e_{j}\right|^{p(t)+1} \\
& \quad+\gamma_{i, 1}\left(l_{2}, \ldots, l_{i}\right)\left|x_{1}-\widehat{x}_{1}\right|^{p(t)+1}
\end{aligned}
$$

where $b_{3}>0$ is a constant, $c_{3, i}>0$ is a constant dependent on $b_{3}$, and $\gamma_{i, j}>0, j=1, \ldots, i-1$, are constants dependent on $b_{3}, l_{j+1}, \ldots, l_{i}$. Similar to (A.6), it follows from (A.8) that

$$
\begin{aligned}
& -\sum_{i=2}^{n} l_{i} e_{i}\left(\phi_{i-1}-\widehat{\phi}_{i-1}\right) \\
& \leq b_{3} \sum_{i=1}^{n}\left|\xi_{i}\right|^{p(t)+1}+\gamma_{n}\left|e_{n}\right|^{p(t)+1} \\
& \quad+\sum_{j=2}^{n-1} \gamma_{j}\left(l_{j+1}, \ldots, l_{n}\right)\left|e_{j}\right|^{p(t)+1} \\
& \quad+\gamma_{1}\left(l_{2}, \ldots, l_{n}\right)\left|x_{1}-\widehat{x}_{1}\right|^{p(t)+1}
\end{aligned}
$$

where $\gamma_{j}=\sum_{i=j+1}^{n} \gamma_{i, j}\left(l_{j+1}, \ldots, l_{i}\right)+c_{3, j}$ with $c_{2,1}=0, j=$ $1, \ldots, n$, that is, $\gamma_{n}>0$ is a constant dependent on $b_{3}$, and $\gamma_{j}>0, j=1, \ldots, n-1$, are constants dependent on $b_{3}, l_{j+1}, \ldots, l_{n}$.

Proof of Proposition 14. By (11), (22), and (A.2), one has

$$
\begin{aligned}
u= & \sum_{i=1}^{n} \beta_{i \sim n}\left(x_{i}-\widehat{x}_{i}\right)-\sum_{i=1}^{n} \beta_{i \sim n} x_{i} \\
= & -\beta_{n} \xi_{n}+\bar{\sigma}_{1}\left(l_{2}, \ldots, l_{n}\right)\left(x_{1}-\widehat{x}_{1}\right) \\
& +\sum_{i=2}^{n-1} \bar{\sigma}_{i}\left(l_{i+1}, \ldots, l_{n}\right) e_{i}+\bar{\sigma}_{n} e_{n}
\end{aligned}
$$

where $\bar{\sigma}_{i}=\sum_{j=i}^{n} \beta_{j \sim n} l_{(i+1) \sim j}, i=1, \ldots, n$, that is, $\bar{\sigma}_{n}$ is a positive constant, and $\bar{\sigma}_{i}, i=1, \ldots, n-1$, are positive constants dependent on $l_{i+1}, \ldots, l_{n}$. By (A.10) and Lemmas 2 and 3 , we derive

$$
\begin{aligned}
e_{n}[u]^{p(t)} \leq & \left|e_{n}\right||u|^{p(t)} \\
\leq & b_{4}\left|\xi_{n}\right|^{p(t)+1}+\sigma_{n}\left|e_{n}\right|^{p(t)+1} \\
& +\sum_{i=2}^{n-1} \sigma_{i}\left(l_{i+1}, \ldots, l_{n}\right)\left|e_{i}\right|^{p(t)+1} \\
& +\sigma_{1}\left(l_{2}, \ldots, l_{n}\right)\left|x_{1}-\widehat{x}_{1}\right|^{p(t)+1},
\end{aligned}
$$

where $b_{4}>0$ is a constant, $\sigma_{n}>0$ is a constant dependent on $b_{4}$, and $\sigma_{i}>0, i=1, \ldots, n-1$, are constants dependent on $l_{i+1}, \ldots, l_{n}$.

Proof of Proposition 15. By $x_{n+1}^{*}=\beta_{n} \xi_{n}$ and (A.10), one has

$$
\begin{aligned}
u-x_{n+1}^{*}= & \bar{\sigma}_{1}\left(l_{2}, \ldots, l_{n}\right)\left(x_{1}-\widehat{x}_{1}\right) \\
& +\sum_{i=2}^{n-1} \bar{\sigma}_{i}\left(l_{i+1}, \ldots, l_{n}\right) e_{i}+\bar{\sigma}_{n} e_{n}
\end{aligned}
$$

from which, by Lemmas 2-4, we derive

$$
\begin{aligned}
\xi_{n} & \left([u]^{p(t)}-\left[x_{n+1}^{*}\right]^{p(t)}\right) \leq\left|\xi_{n}\right| \cdot \bar{p}\left(2^{\bar{p}-3}+1\right) \\
\cdot & \left(\left|u-x_{n+1}^{*}\right|^{p(t)}+\left|u-x_{n+1}^{*}\right|\left|x_{n+1}^{*}\right|^{p(t)-1}\right) \\
& \leq b_{5}\left|\xi_{n}\right|^{p(t)+1}+\omega_{n}\left|e_{n}\right|^{p(t)+1} \\
& +\sum_{i=2}^{n-1} \omega_{i}\left(l_{i+1}, \ldots, l_{n}\right)\left|e_{i}\right|^{p(t)+1}+\omega_{1}\left(l_{2}, \ldots, l_{n}\right) \\
& \cdot\left|x_{1}-\widehat{x}_{1}\right|^{p(t)+1},
\end{aligned}
$$

where $b_{5}>0$ is a constant, $\omega_{n}>0$ is a constant dependent on $b_{5}$, and $\omega_{i}>0, i=1, \ldots, n-1$, are constants dependent on $b_{5}, l_{i+1}, \ldots, l_{n}$.

\section{Data Availability}

The data supporting the conclusions of the manuscript are some open access articles that have been properly cited, and the readers can easily obtain these articles to verify the conclusions, replicate the analysis, and conduct secondary analyses. Therefore, we do not create a publicly available data repository.

\section{Conflicts of Interest}

The authors declare that they have no competing interests.

\section{Acknowledgments}

This work was supported by the Taishan Scholar Project of Shandong Province of China (No. ts201712040), National Natural Science Foundation of China (No. 61673242), and Shandong Provincial Natural Science Foundation of China (No. ZR2016FM10). 


\section{References}

[1] W. Lin and C.-J. Qian, "Adding one power integrator: a tool for global stabilization of high-order lower-triangular systems," Systems \& Control Letters, vol. 39, no. 5, pp. 339-351, 2000.

[2] W. Lin, C.-J. Qian, and X.-Q. Huang, "Disturbance attenuation of a class of non-linear systems via output feedback," International Journal of Robust and Nonlinear Control, vol. 13, no. 15, pp. 1359-1369, 2003.

[3] B. Yang and W. Lin, "Homogeneous observers, iterative design, and global stabilization of high-order nonlinear systems by smooth output feedback," Institute of Electrical and Electronics Engineers Transactions on Automatic Control, vol. 49, no. 7, pp. 1069-1080, 2004.

[4] J. Polendo and C.-J. Qian, "A generalized homogeneous domination approach for global stabilization of inherently nonlinear systems via output feedback," International Journal of Robust and Nonlinear Control, vol. 17, no. 7, pp. 605-629, 2007.

[5] X.-J. Xie and X.-H. Zhang, "Further results on global state feedback stabilization of nonlinear high-order feedforward systems," ISA Transactions ${ }^{\circledR}$, vol. 53, no. 2, pp. 341-346, 2014.

[6] X.-H. Zhang and X.-J. Xie, "Global state feedback stabilisation of nonlinear systems with high-order and low-order nonlinearities," International Journal of Control, vol. 87, no. 3, pp. 642-652, 2014.

[7] X.-J. Xie, N. Duan, and C.-R. Zhao, "A combined homogeneous domination and sign function approach to output-feedback stabilization of stochastic high-order nonlinear systems," IEEE Transactions on Automatic Control, vol. 59, no. 5, pp. 1303-1309, 2014.

[8] Z.-Q. Zhang, S.-Y. Xu, and B.-Y. Zhang, "Asymptotic tracking control of uncertain nonlinear systems with unknown actuator nonlinearity," IEEE Transactions on Automatic Control, vol. 59, no. 5, pp. 1336-1341, 2014.

[9] X.-H. Zhang, X.-J. Xie, and Y.-X. Huang, "Global state feedback stabilization of high-order nonlinear systems with multiple time-varying delays," Journal of The Franklin Institute, vol. 352, no. 1, pp. 271-290, 2015.

[10] Z.-Y. Sun, L.-R. Xue, and K.-M. Zhang, "A new approach to finite-time adaptive stabilization of high-order uncertain nonlinear system," Automatica, vol. 58, no. 8, pp. 60-66, 2015.

[11] K.-M. Zhang and X.-H. Zhang, "Finite-time stabilisation for high-order nonlinear systems with low-order and high-order nonlinearities," International Journal of Control, vol. 88, no. 8, pp. 1576-1585, 2015.

[12] X.-H. Zhang, K.-M. Zhang, and M.-M. Jiang, "Global output feedback stabilisation of high-order nonlinear systems with low-order and high-order nonlinearities," International Journal of Control, vol. 88, no. 11, pp. 2194-2210, 2015.

[13] Z.-Y. Sun, T. Li, and S.-H. Yang, "A unified time-varying feedback approach and its applications in adaptive stabilization of high-order uncertain nonlinear systems," Automatica, vol. 70, no. 8, pp. 249-257, 2016.

[14] X.-H. Zhang, K.-M. Zhang, and X.-J. Xie, "Finite-time output feedback stabilization of nonlinear high-order feedforward systems," International Journal of Robust and Nonlinear Control, vol. 26, no. 8, pp. 1794-1814, 2016.

[15] Z.-Y. Sun, C.-H. Zhang, and Z. Wang, "Adaptive disturbance attenuation for generalized high-order uncertain nonlinear systems," Automatica, vol. 80, pp. 102-109, 2017.
[16] Z.-Y. Sun, M.-M. Yun, and T. Li, "A new approach to fast global finite-time stabilization of high-order nonlinear system," Automatica, vol. 81, pp. 455-463, 2017.

[17] J.-Y. Zhai and C.-J. Qian, "Global control of nonlinear systems with uncertain output function using homogeneous domination approach," International Journal of Robust and Nonlinear Control, vol. 22, no. 14, pp. 1543-1561, 2012.

[18] W.-Q. Ai, J.-Y. Zhai, and S.-M. Fei, "Universal adaptive regulation for a class of nonlinear systems with unknown time delays and output function via output feedback," Journal of The Franklin Institute, vol. 350, no. 10, pp. 3168-3187, 2013.

[19] M.-M. Jiang, P. Wang, and X.-J. Xie, “Global output feedback stabilisation of nonlinear time-delay systems with unknown output function," International Journal of Systems Science, vol. 48, no. 12, pp. 2554-2564, 2017.

[20] J.-Y. Zhai, "Global finite-time output feedback stabilisation for a class of uncertain nontriangular nonlinear systems," International Journal of Systems Science, vol. 45, no. 3, pp. 637646, 2014.

[21] X.-J. Xie, Z.-J. Li, and K.-M. Zhang, "Semi-global output feedback control for nonlinear systems with uncertain timedelay and output function," International Journal of Robust and Nonlinear Control, vol. 27, no. 15, pp. 2549-2566, 2017.

[22] Z.-J. Li, X.-J. Xie, and K.-M. Zhang, "Output feedback stabilisation for nonlinear systems with unknown output function and control coefficients and its application," International Journal of Control, vol. 90, no. 5, pp. 1027-1036, 2017.

[23] M.-M. Jiang, X.-J. Xie, and K.-M. Zhang, "Finite-time output feedback stabilization of high-order uncertain nonlinear systems," International Journal of Control, vol. 91, no. 6, pp. 13381349, 2018.

[24] C.-L. Rui, M. Reyhanoglu, I. Kolmanovsky, S. Cho, and N.H. McClamroch, "Nonsmooth stabilization of an underactuated unstable two degrees of freedom mechanical system," in Proceedings of the 1997 36th IEEE Conference on Decision and Control, vol. 4, pp. 3998-4003, San Diego, Calif, USA, December 1997.

[25] J.-Z. Liu, S. Yan, D.-L. Zeng, Y. Hu, and Y. Lv, "A dynamic model used for controller design of a coal fired once-through boilerturbine unit," Energy, vol. 93, pp. 2069-2078, 2015.

[26] Z.-G. Su, C.-J. Qian, and J. Shen, "Interval homogeneitybased control for a class of nonlinear systems with unknown power drifts," Institute of Electrical and Electronics Engineers Transactions on Automatic Control, vol. 62, no. 3, pp. 1445-1450, 2017.

[27] C.-C. Chen, C.-J. Qian, Y.-W. Liang, and S.-H. Li, "Interval homogeneous domination approach for global stabilization of nonlinear systems with time-varying powers," in Proceedings of the 55th IEEE Conference on Decision and Control, CDC 2016, pp. 7258-7263, USA, December 2016.

[28] C.-C. Chen and S. S.-D. Xu, "Global Stabilization for a Class of Genuinely Nonlinear Systems with a Time-Varying Power: An Interval Homogeneous Domination Approach," IEEE Access, vol. 6, pp. 11255-11264, 2018.

[29] C.-C. Chen, C.-J. Qian, X.-Z. Lin, Z.-Y. Sun, and Y.-W. Liang, "Smooth output feedback stabilization for a class of nonlinear systems with time-varying powers," International Journal of Robust and Nonlinear Control, vol. 27, no. 18, pp. 5113-5128, 2017.

[30] H.-K. Khalil, Nonlinear Systems, Prentice-Hall, New Jersey, NJ, USA, 2002.

[31] G. Hardy, J. Littlewood, and G. Polya, Inequalities, Cambridge University Press, Cambridge, UK, 2nd edition, 1988. 
[32] Z.-Y. Sun, X.-H. Zhang, and X.-J. Xie, "Continuous global stabilisation of high-order time-delay nonlinear systems," International Journal of Control, vol. 86, no. 6, pp. 994-1007, 2013.

[33] Z.-Y. Sun, X.-H. Zhang, and X.-J. Xie, "Global continuous output-feedback stabilization for a class of high-order nonlinear systems with multiple time delays," Journal of The Franklin Institute, vol. 351, no. 8, pp. 4334-4356, 2014. 


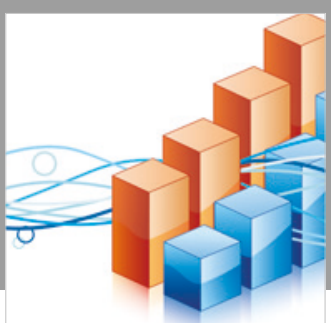

Advances in

Operations Research

\section{-n-m}
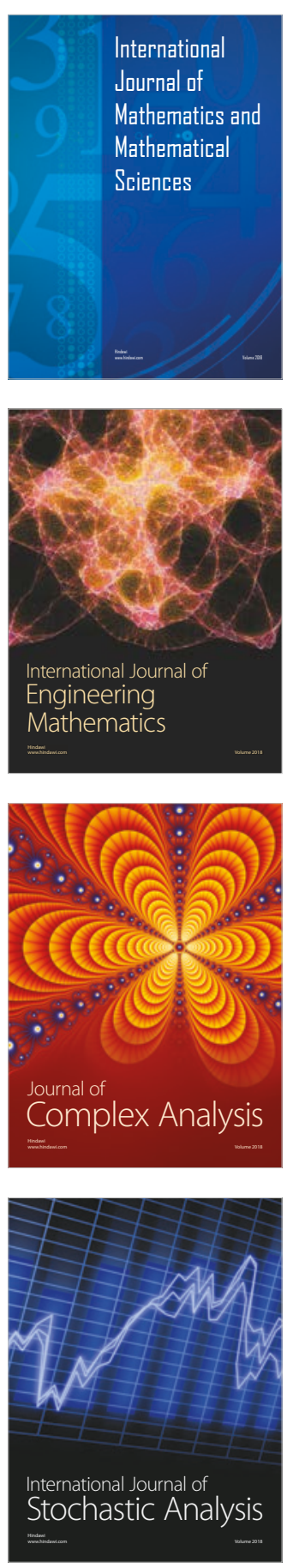
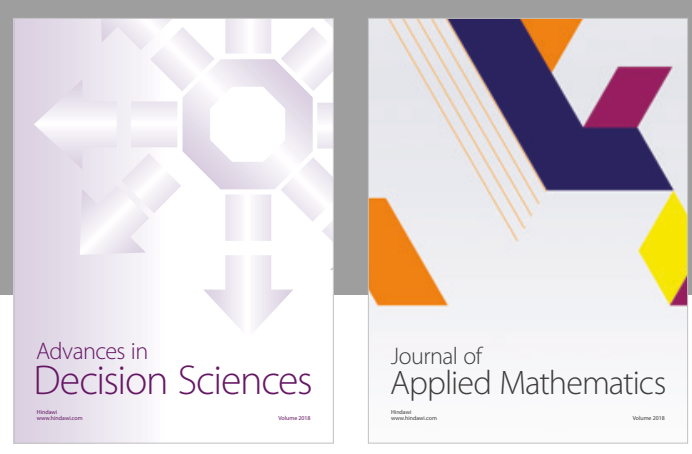

Journal of

Applied Mathematics
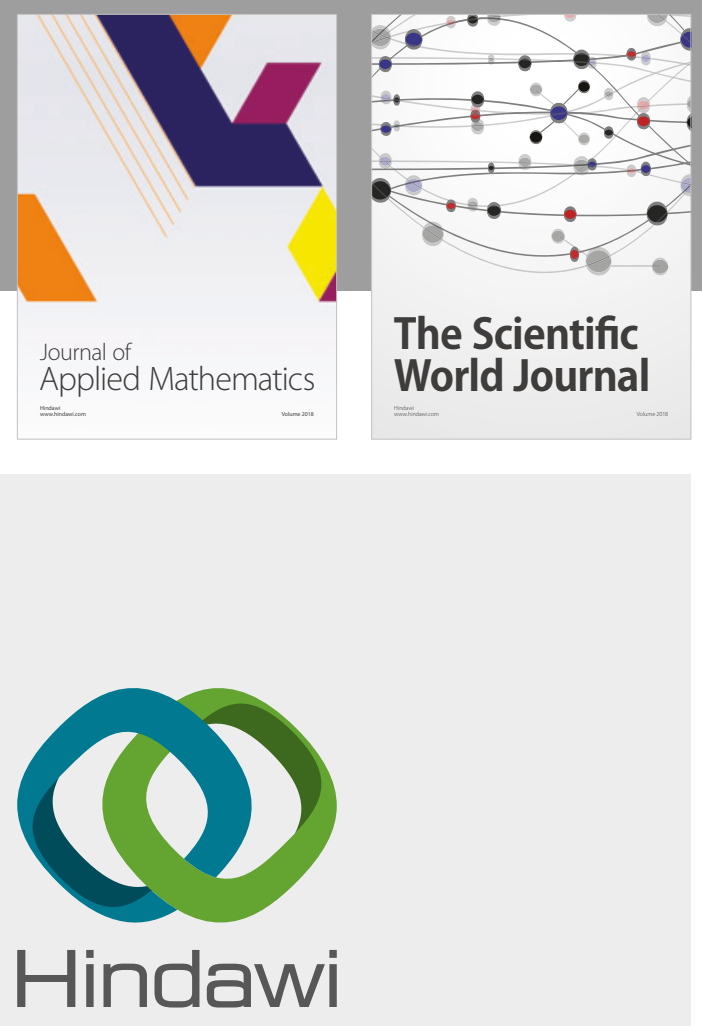

Submit your manuscripts at

www.hindawi.com

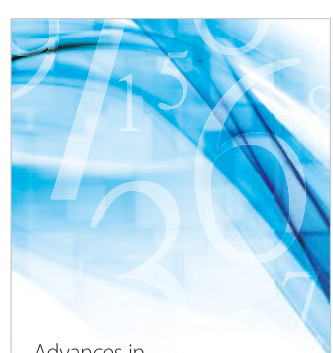

Advances in
Numerical Analysis
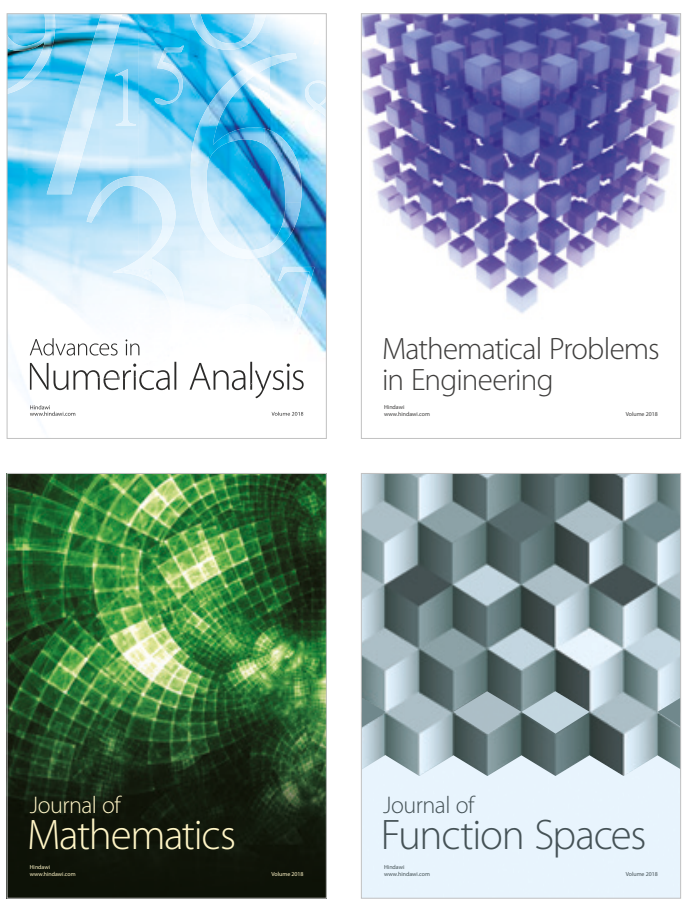

Mathematical Problems in Engineering

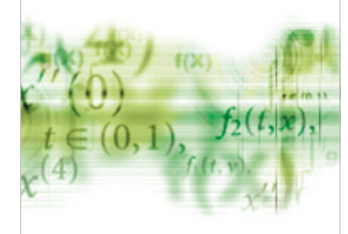

International Journal of

Differential Equations

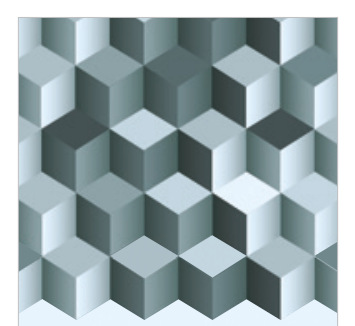

Journal of

Function Spaces

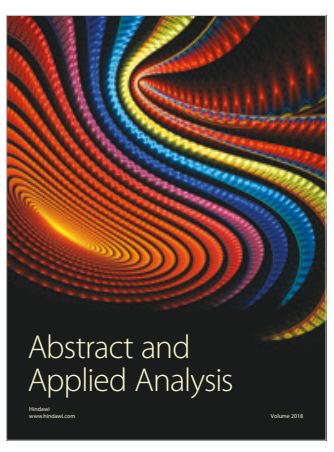

The Scientific

World Journal

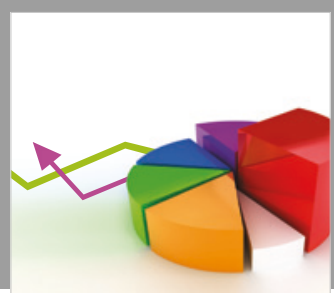

Journal of

Probability and Statistics
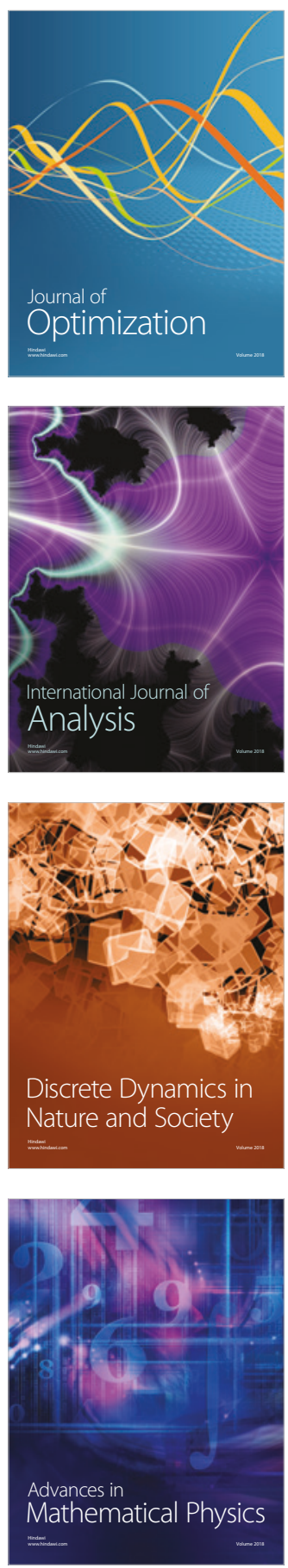